\title{
Efficient Pricing of European-Style Options under Heston's Stochastic Volatility Model
}

\author{
Oleksandr Zhylyevskyy \\ Department of Economics, Iowa State University, Ames, IA, USA \\ Email: oz9a@iastate.edu
}

Received November 10, 2011; revised December 24, 2011; accepted December 30, 2011

\begin{abstract}
Heston's stochastic volatility model is frequently employed by finance researchers and practitioners. Fast pricing of European-style options in this setting has considerable practical significance. This paper derives a computationally efficient formula for the value of a European-style put under Heston's dynamics, by utilizing a transform approach based on inverting the characteristic function of the underlying stock's log-price and by exploiting the characteristic function's symmetry. The value of a European-style call is computed using a parity relationship. The required characteristic function is obtained as a special case of a more general solution derived in prior research. Computational advantage of the option value formula is illustrated numerically. The method can help to mitigate the time cost of algorithms that require repeated evaluation of European-style options under Heston's dynamics.
\end{abstract}

Keywords: Heston's Model; European-Style Option; Characteristic Function Inversion

\section{Introduction}

Stochastic volatility models such as the model of Heston (1993) [1] are a frequent choice among finance researchers and practitioners to approximate stock price dynamics. Popularity of Heston's model stems from its ability to reproduce a number of important time-series features of stock prices that are not adequately captured by geometric Brownian motion dynamics. In particular, Heston's model is able to account for the leverage effect, that is, an increase in the volatility of a stock when its price declines, and a decrease in the volatility when the price rises (for more details, see Aït-Sahalia and Kimmel, 2007 [2]). As such, the model is often employed when pricing derivative securities, since it helps to correct for empirical deficiencies of the famous Black and Scholes' (1973) [3] formula (for a discussion, see Aït-Sahalia and Lo, 1998 [4]).

Under Heston's dynamics, the values of Europeanstyle options cannot be expressed in closed form. Instead, they may be computed numerically using the transform methods of Duffie et al. (2000) [5] and Bakshi and Madan (2000) [6], which require inverting the characteristic function of an underlying state price vector. The characteristic function can be expressed analytically using

\footnotetext{
${ }^{1}$ In recent years, there has been substantial interest in pricing Americanstyle options under Heston's dynamics with a finite-difference method, which has presented researchers with many challenges (e.g., Zvan et al., 1998 [9]; Clarke and Parrott, 1999 [10]; Oosterlee, 2003 [11]; Ikonen and Toivanen, 2007 [12]).
}

Heston's original solution, or alternatively obtained as a special case of a more general solution of Zhylyevskyy (2010) [7]. This paper applies a particularly suitable inversion procedure and exploits symmetry properties of characteristic functions, in order to derive a computationally efficient formula for the value of a European-style put. The value of a European-style call is obtained using a parity relationship for European-style derivatives. Exploiting the symmetry of the characteristic function allows for a reduction of the domain of integration in the inversion, and cuts roughly in half the time needed for option valuation.

Efficient pricing of European-style options under Heston's dynamics is important from a practical standpoint, since many single-name and index equity options listed on organized exchanges are European-style. Also, values of the European-style options may be used in methods of calculating prices of derivative securities of a different style, for example, in the Geske-Johnson method (Geske and Johnson, 1984 [8]) of pricing American-style options using a compound-option technique (for an implementation, see Zhylyevskyy, 2010 [7]). ${ }^{1}$

\section{Stock Price Dynamics}

The financial market is assumed to admit no arbitrage opportunities. Thus, there is a risk-neutral probability measure, denoted here as $P$, under which discounted asset prices are martingales (Harrison and Kreps, 1979 [13]). 
One of the traded assets is a riskless bond fund with a share worth $M_{t}=M_{0} \mathrm{e}^{r t}$ at time $t$, where $M_{0}>0$ is some initial value and $r \geq 0$ is a risk-free rate. It will be convenient to use this bond fund as a numéraire security and discount asset prices by $M_{t}$ when appropriate.

The dynamics of a stock price, $S_{t}$, under $P$ are described by a system of stochastic differential equations

$$
\begin{aligned}
& \mathrm{d} S_{t}=(r-\delta) S_{t} \mathrm{~d} t+\sqrt{v_{t}} S_{t} \mathrm{~d} W_{1 t}, \\
& \mathrm{~d} v_{t}=\left(\alpha-\beta v_{t}\right) \mathrm{d} t+\gamma \sqrt{v_{t}} \mathrm{~d} W_{2 t},
\end{aligned}
$$

where $\delta \geq 0$ stands for a dividend rate, $v_{t}$ represents an unobservable squared volatility, $W_{1 t}$ and $W_{2 t}$ are correlated standard Brownian motions on a filtered probability space $\left(\Omega, \mathcal{F},\left\{\mathcal{F}_{t}\right\}_{t \geq 0}, P\right)$ with $d\left\langle W_{1}, W_{2}\right\rangle_{t}=\rho \mathrm{d} t$ and $|\rho|<1$, and $\alpha, \beta$, and $\gamma$ are nonnegative constants satisfying a restriction $\gamma^{2} \leq 2 \alpha$ in order for $v_{t}$ to be almost surely positive (Chernov and Ghysels, 2000 [14]).

To derive values of European-style options, it will be convenient to work with a $\log$-price, $s_{t}=\ln S_{t}$. Applying Ito's lemma to Equation (1), the evolution of the logprice is described by a stochastic differential equation

$$
\mathrm{d} s_{t}=\left(r-\delta-v_{t} / 2\right) \mathrm{d} t+\sqrt{v_{t}} \mathrm{~d} W_{1 t} .
$$

\section{Characteristic Function}

Let $\tau$ denote the time duration between current date $t$ and some future date $T, \tau=T-t$. Given the log-price $s_{t}$ and squared volatility $v_{t}$, the conditional characteristic function of the state vector $\left(s_{T}, v_{T}\right)$ is

$$
\psi\left(\zeta_{1}, \zeta_{2} ; s_{t}, v_{t}, \tau\right)=E\left[\mathrm{e}^{i\left(\zeta_{1} s_{T}+\zeta_{2} v_{T}\right)} \mid \mathcal{F}_{t}\right]
$$

where $E$ is the expected value taken under $P$ and $\zeta_{1}$ and $\zeta_{2}$ are real-valued arguments.

Likewise, the conditional characteristic function of $\left(s_{T}, v_{T}\right)$ at a date $u \in(t, T]$ is

$$
\psi\left(\zeta_{1}, \zeta_{2} ; s_{u}, v_{u}, T-u\right)=E\left[\mathrm{e}^{i\left(\zeta_{1} s_{T}+\zeta_{2} v_{T}\right)} \mid \mathcal{F}_{u}\right]
$$

Since $u>t$, the $\sigma$-field $\mathcal{F}_{t} \subset \mathcal{F}_{u}$, and therefore,

$$
E\left[\mathrm{e}^{i\left(\zeta_{1} s_{T}+\zeta_{2} v_{T}\right)} \mid \mathcal{F}_{t}\right]=E\left[E\left[\mathrm{e}^{i\left(\zeta_{1} s_{T}+\zeta_{2} v_{T}\right)} \mid \mathcal{F}_{u}\right] \mid \mathcal{F}_{t}\right]
$$

by the law of iterated expectations. Thus,

$$
E\left[\mathrm{~d} \psi\left(\zeta_{1}, \zeta_{2} ; s_{t}, v_{t}, \tau\right) \mid \mathcal{F}_{t}\right]=0
$$

by taking $u$ arbitrarily close to $t$. Applying Ito's lemma to $\psi$ it follows that

$$
\begin{aligned}
\mathrm{d} \psi\left(\zeta_{1}, \zeta_{2} ; s_{t}, v_{t}, \tau\right)= & -\psi_{\tau} \mathrm{d} t+\psi_{s} \mathrm{~d} s_{t}+\psi_{v} \mathrm{~d} v_{t}+\psi_{s s} \mathrm{~d}\langle s\rangle_{t} / 2 \\
& +\psi_{v v} \mathrm{~d}\langle v\rangle_{t} / 2+\psi_{s v} \mathrm{~d}\langle s, v\rangle_{t},
\end{aligned}
$$

where $\psi_{x}$ and $\psi_{x y}$ denote $\partial \psi / \partial x$ and $\partial^{2} \psi / \partial x \partial y$, respectively. Then, Equations (2), (3), and (4) imply that $\psi$ solves a partial differential equation

$$
\begin{aligned}
& -\psi_{\tau}+\psi_{s}\left(r-\delta-v_{t} / 2\right)+\psi_{v}\left(\alpha-\beta v_{t}\right) \\
& +\psi_{s s} v_{t} / 2+\psi_{v v} \gamma^{2} v_{t} / 2+\psi_{s v} \rho \gamma v_{t}=0 .
\end{aligned}
$$

Zhylyevskyy (2010) [7] provides a complete solution for $\psi$ using Equation (5). For reference, the solution is outlined in the appendix. The marginal conditional characteristic function of the $\log$-price $s_{T}$, which will be inverted to derive the value of a European-style put, is obtained as a special case:

$$
\varphi\left(\zeta ; s_{t}, v_{t}, \tau\right)=\psi\left(\zeta, 0 ; s_{t}, v_{t}, \tau\right),
$$

where $\zeta$ is a real-valued argument.

The complex-valued function $\varphi(\zeta)$ is symmetric in the sense that its real part is an even function,

$$
\operatorname{Re}[\varphi(-\zeta)]=\operatorname{Re}[\varphi(\zeta)],
$$

while its imaginary part is an odd function,

$$
\operatorname{Im}[\varphi(-\zeta)]=-\operatorname{Im}[\varphi(\zeta)] \text {. }
$$

This symmetry will allow for a simplification of the option value's expression and is a property of all characteristic functions, as Lemma 1 shows.

Lemma 1. Let $\phi(\zeta)$ be the characteristic function of a random vector $Y$, where $\zeta$ is a real-valued vector of the same dimension as $Y$. The real part of $\phi(\zeta)$ is an even function,

$$
\operatorname{Re}[\phi(-\zeta)]=\operatorname{Re}[\phi(\zeta)]
$$

and the imaginary part of $\phi(\zeta)$ is an odd function,

$$
\operatorname{Im}[\phi(-\zeta)]=-\operatorname{Im}[\phi(\zeta)] .
$$

Proof. By definition, $\phi(\zeta)=\int \mathrm{e}^{i \zeta^{\prime} Y} \mathrm{~d} F$, where $F$ is the cumulative distribution function of $Y$. Applying Euler's formula,

and

$$
\begin{aligned}
& \operatorname{Re}[\phi(\zeta)]=\int \cos \left(\zeta^{\prime} Y\right) \mathrm{d} F \\
& \operatorname{Im}[\phi(\zeta)]=\int \sin \left(\zeta^{\prime} Y\right) \mathrm{d} F .
\end{aligned}
$$

It then follows from the properties of trigonometric functions that

and

$$
\begin{aligned}
& \operatorname{Re}[\phi(-\zeta)]=\operatorname{Re}[\phi(\zeta)] \\
& \operatorname{Im}[\phi(-\zeta)]=-\operatorname{Im}[\phi(\zeta)] .
\end{aligned}
$$

\section{Option Value Formulas}

This section applies the transform methods of Duffie et al. (2000) [5] and Bakshi and Madan (2000) [6], and exploits the symmetry of the characteristic function $\varphi$ to obtain computationally efficient formulas for values of European-style puts and calls under Heston's dynamics. Initial steps of the derivation follow the approach of Epps (2004) [15], but the idea to exploit the symmetry of the characteristic function is novel. 
Let $P^{E}\left(X, S_{t}, v_{t}, \tau\right)$ denote the current value of a European-style put with a strike price $X$, given the (current) underlying stock's price $S_{t}$, volatility $v_{t}$, and time to expiration $\tau$. The put is allowed to be exercised on date $T$ when its value will be $P^{E}\left(X, S_{T}, v_{T}, 0\right)=$ $\max \left\{0, X-S_{T}\right\}$. The first fundamental theorem of asset pricing implies that the put's value, discounted by the share price of the riskless bond fund, is a martingale. Thus,

$$
M_{t}^{-1} P^{E}\left(X, S_{t}, v_{t}, \tau\right)=E\left[M_{T}^{-1} P^{E}\left(X, S_{T}, v_{T}, 0\right) \mid \mathcal{F}_{t}\right],
$$

and therefore,

$$
\begin{aligned}
P^{E}\left(X, S_{t}, v_{t}, \tau\right) & =\mathrm{e}^{-r \tau} E\left[\max \left\{0, X-S_{T}\right\} \mid \mathrm{F}_{t}\right] \\
& =\mathrm{e}^{-r \tau} \int_{0}^{X}(X-S) \mathrm{d} F(S),
\end{aligned}
$$

where $F$ is the conditional cumulative distribution function of $S_{T}$. Note that $F(0)=0$, since the stock price cannot be negative. Integrating $\int_{0}^{X} S \mathrm{~d} F$ by parts, the put's value can be expressed as

$$
\begin{aligned}
P^{E}\left(X, S_{t}, v_{t}, \tau\right) & =\mathrm{e}^{-r \tau}\left[X F(X)-\int_{0}^{X} S \mathrm{~d} F(S)\right] \\
& =\mathrm{e}^{-r \tau} \int_{0}^{X} F(S) \mathrm{d} S .
\end{aligned}
$$

Observe that

$$
F(S)=\operatorname{Pr}\left[S_{T} \leq S \mid \mathcal{F}_{t}\right]=\operatorname{Pr}\left[s_{T} \leq \ln S \mid \mathcal{F}_{t}\right]=G(\ln S),
$$

where $G$ is the conditional cumulative distribution function of the log-price $s_{T}$. Hence, by changing variables as $s=\ln S$, the put's value can be re-expressed as

$$
P^{E}\left(X, S_{t}, v_{t}, \tau\right)=\mathrm{e}^{-r \tau} \int_{-\infty}^{\ln X} G(s) \mathrm{e}^{s} \mathrm{~d} s .
$$

The characteristic function $\varphi$ can be inverted to obtain $G$ as follows (Gil-Pelaez, 1951 [16]):

$$
G(s)=\frac{1}{2}-\frac{1}{2 \pi} \int_{-\infty}^{\infty} \frac{\mathrm{e}^{-i \zeta s}}{i \zeta} \varphi\left(\zeta ; s_{t}, v_{t}, \tau\right) \mathrm{d} \zeta .
$$

Plugging Equation (8) into Equation (7),

$$
\begin{aligned}
& P^{E}\left(X, S_{t}, v_{t}, \tau\right) \\
= & \mathrm{e}^{-r \tau} \int_{-\infty}^{\ln X}\left[\frac{1}{2}-\frac{1}{2 \pi} \int_{-\infty}^{\infty} \frac{\mathrm{e}^{-i \zeta s}}{i \zeta} \varphi\left(\zeta ; s_{t}, v_{t}, \tau\right) \mathrm{d} \zeta\right] \mathrm{e}^{s} \mathrm{~d} s \\
= & \mathrm{e}^{-r \tau}\left[\frac{X}{2}-\frac{1}{2 \pi} \int_{-\infty}^{\infty} \int_{-\infty}^{\ln X} \frac{\mathrm{e}^{s(1-i \zeta)}}{i \zeta} \varphi\left(\zeta ; s_{t}, v_{t}, \tau\right) \mathrm{d} s \mathrm{~d} \zeta\right] \\
= & \frac{\mathrm{e}^{-r \tau} X}{2}\left[1-\frac{1}{\pi} \int_{-\infty}^{\infty} \frac{X^{-i \zeta}}{i \zeta+\zeta^{2}} \varphi\left(\zeta ; s_{t}, v_{t}, \tau\right) \mathrm{d} \zeta\right],
\end{aligned}
$$

where the change in the integration order after the second equality sign is due to Fubini's theorem. ${ }^{2}$

\footnotetext{
${ }^{2}$ Observe that the double integral is finite because

$P^{E}\left(X, S_{t}, v_{t}, \tau\right)=\mathrm{e}^{-r \tau} E\left[\max \left\{0, X-S_{T}\right\} \mid \mathcal{F}_{t}\right] \leq \mathrm{e}^{-r \tau} E\left[X \mid \mathcal{F}_{t}\right]=\mathrm{e}^{-r \tau} X<\infty$.
}

The put option value formula, Equation (9), can be simplified by exploiting the symmetry of $\varphi$. Denoting the complex-valued integrand in Equation (9) as $\Lambda(\zeta)$ and applying Euler's formula to it,

$$
\begin{aligned}
\Lambda(\zeta)= & \frac{X^{-i \zeta}}{i \zeta+\zeta^{2}} \varphi(\zeta) \\
= & \frac{\left(\zeta^{2}-i \zeta\right)(\cos (\zeta \ln X)-i \sin (\zeta \ln X))}{\left(\zeta^{2}+i \zeta\right)\left(\zeta^{2}-i \zeta\right)} \\
& \times(\operatorname{Re}[\varphi(\zeta)]+i \min [\varphi(\zeta)]) \\
= & \frac{(\zeta \cos (\zeta \ln X)-\sin (\zeta \ln X)) \operatorname{Re}[\varphi(\zeta)]}{\zeta^{3}+\zeta} \\
& +\frac{(\zeta \sin (\zeta \ln X)+\cos (\zeta \ln X)) \operatorname{Im}[\varphi(\zeta)]}{\zeta^{3}+\zeta} \\
& +i \frac{(\zeta \cos (\zeta \ln X)-\sin (\zeta \ln X)) \operatorname{Im}[\varphi(\zeta)]}{\zeta^{3}+\zeta} \\
& -i \frac{(\zeta \sin (\zeta \ln X)+\cos (\zeta \ln X)) \operatorname{Re}[\varphi(\zeta)]}{\zeta^{3}+\zeta} .
\end{aligned}
$$

It then follows from the properties of trigonometric functions and Lemma 1 that $\operatorname{Re}[\Lambda(-\zeta)]=\operatorname{Re}[\Lambda(\zeta)]$ and $\operatorname{Im}[\Lambda(-\zeta)]=-\operatorname{Im}[\Lambda(\zeta)]$. Therefore, by applying properties of even and odd functions,

$$
\begin{aligned}
& P^{E}\left(X, S_{t}, v_{t}, \tau\right) \\
= & \frac{\mathrm{e}^{-r \tau} X}{2} \times\left[1-\frac{2}{\pi} \int_{0}^{\infty} \operatorname{Re}\left[\frac{X^{-i \zeta}}{i \zeta+\zeta^{2}} \varphi\left(\zeta ; s_{t}, v_{t}, \tau\right)\right] \mathrm{d} \zeta\right] .
\end{aligned}
$$

The oddness property of the imaginary part of $\Lambda$ is not particularly surprising, since the imaginary part must integrate out to zero in order for $P^{E}\left(X, S_{t}, v_{t}, \tau\right)$ in Equation (9) to be real-valued. However, the evenness property of the real part of $\Lambda$ is not immediately obvious. It allows for a reduction of the domain of integration, as Equation (10) shows.

Finally, let $C^{E}\left(X, S_{t}, v_{t}, \tau\right)$ denote the current value of a European-style call with a strike price $X$, given the stock's price $S_{t}$, volatility $v_{t}$, and time to expiration $\tau$. The call is allowed to be exercised on date $T$ when its value will be $C^{E}\left(X, S_{T}, v_{T}, 0\right)=\max \left\{0, S_{T}-X\right\}$. By a put-call parity relationship for European-style options (Merton, 1973 [17]), which holds regardless of specific stock price dynamics,

$$
C^{E}\left(X, S_{t}, v_{t}, \tau\right)=P^{E}\left(X, S_{t}, v_{t}, \tau\right)+\mathrm{e}^{-\delta \tau} S_{t}-\mathrm{e}^{-r \tau} X,
$$

where $P^{E}\left(X, S_{t}, v_{t}, \tau\right)$ can be obtained using Equation (10).

\section{Numerical Investigation}

Equations (9) and (10) require numerical integration to 
calculate option values in practice. Intuitively, reducing the domain of integration in Equation (10) should allow for an increase in computational speed without a loss in precision. To assess actual performance of the two option value formulas, I conduct a numerical exercise. Namely, I calculate large series of put option values by applying both Equation (9) and Equation (10), and record the total computation time in each case. Parameter values used in the exercise are listed in Table 1 and correspond to calibrated values for the dynamics of the S \& P 100 Index in July 2004 (Zhylyevskyy, 2010 [7]). ${ }^{3}$ Series of put options are generated by varying the underlying price $S_{t}$ from 450 to 550 and the strike price $X$ from 350 to 650 . All options have a duration of three months. Numerical integration is implemented using the Gauss-Kronrod quadrature method (Press et al., 2001 [18]).

Table 2 reports the ratio of time needed to calculate all option values in series comprising 100, 10,000, and 1,000,000 options, using Equation (9) relative to Equation (10). As can be seen, by applying Equation (10) instead of Equation (9), the computation time can be cut almost in half. This outcome is expected since the integration in Equation (9) is conducted over the entire real line, while in Equation (10) it is only performed over the nonnegative domain. Most likely, since a small fixed cost of calling the integration routine is borne regardless of the region of integration, the computation speed increases by slightly less than twice overall.

The results of the numerical exercise indicate a computational advantage of Equation (10). This option value formula can help to reduce the cost of computationally intensive tasks that require evaluating multiple European-style derivative securities under Heston's dynamics, such as in the cases of calibrating model parameters and calculating American-style option values by the compound-option scheme of Geske and Johnson (1984) [8].

Table 1. Parameter values in numerical exercise.

\begin{tabular}{cccc}
\hline Parameter & Value & Parameter & Value \\
\hline$\alpha$ & 0.3539 & $v_{t}$ & 0.0114 \\
$\beta$ & 9.5613 & $r$ & 0.0100 \\
$\gamma$ & 0.7637 & $\delta$ & 0.0300 \\
$\rho$ & -0.6924 & & \\
\hline
\end{tabular}

Table 2. Relative performance of option value formulas.

\begin{tabular}{cc}
\hline Number of Options & Time Ratio \\
\hline 100 & 1.96 \\
10,000 & 1.98 \\
$1,000,000$ & 1.95 \\
\hline
\end{tabular}

\footnotetext{
${ }^{3}$ The S \& P 100 Index includes 100 leading stocks in the United States, which are components of the broader S \& P 500 Composite Index and represent roughly $45 \%$ of the equity market capitalization.
}

\section{Conclusion}

Efficient pricing of European-style derivatives under the stochastic volatility dynamics of Heston's model has practical relevance, due to the model's popularity. This paper utilizes a standard transform approach based on inverting the characteristic function of the log-price, and exploits the function's symmetry in order to derive a computationally efficient formula for the value of a European-style put. The value of a call option is obtained using the parity relationship. The computational advantage of the formula stems from reducing the domain of integration in the inversion procedure, and is illustrated numerically. The method can help to mitigate the time cost of algorithms that require repeated evaluation of European-style options under Heston's dynamics.

\section{REFERENCES}

[1] S. L. Heston, "A Closed-Form Solution for Options with Stochastic Volatility with Applications to Bond and Currency Options," Review of Financial Studies, Vol. 6, No. 2, 1993, pp. 327-343. doi:10.1093/rfs/6.2.327

[2] Y. Ait-Sahalia and R. Kimmel, "Maximum Likelihood Estimation of Stochastic Volatility Models," Journal of Financial Economics, Vol. 83, No. 2, 2007, pp. 413-452. doi:10.1016/j.jfineco.2005.10.006

[3] F. Black and M. Scholes, "The Pricing of Options and Corporate Liabilities," Journal of Political Economy, Vol. 81, No. 3, 1973, pp. 637-654. doi:10.1086/260062

[4] Y. Aït-Sahalia and A. W. Lo, "Nonparametric Estimation of State-Price Densities Implicit in Financial Asset Prices," Journal of Finance, Vol. 53, No. 2, 1998, pp. 499-547. doi:10.1111/0022-1082.215228

[5] D. Duffie, J. Pan and K. Singleton, "Transform Analysis and Asset Pricing for Affine Jump-Diffusions," Econometrica, Vol. 68, No. 6, 2000, pp. 1343-1376. doi:10.1111/1468-0262.00164

[6] G. Bakshi and D. Madan, "Spanning and DerivativeSecurity Valuation," Journal of Financial Economics, Vol. 55, No. 3, 2000, pp. 205-238. doi:10.1016/S0304-405X(99)00050-1

[7] O. Zhylyevskyy, "A Fast Fourier Transform Technique for Pricing American Options under Stochastic Volatility," Review of Derivatives Research, Vol. 13, No. 1, 2010, pp. 1-24. doi:10.1007/s11147-009-9041-6

[8] R. Geske and H. E. Johnson, "The American Put Option Valued Analytically," Journal of Finance, Vol. 39, No. 5, 1984, pp. 1511-1524. doi:10.2307/2327741

[9] R. Zvan, P. A. Forsyth and K. R. Vetzal, "Penalty Methods for American Options with Stochastic Volatility," Journal of Computational and Applied Mathematics, Vol. 91, No. 4, 1998, pp. 199-218. doi:10.1016/S0377-0427(98)00037-5

[10] N. Clarke and K. Parrott, "Multigrid for American Option Pricing with Stochastic Volatility," Applied Mathematical Finance, Vol. 6, No. 3, 1999, pp. 177-195. 


\section{doi:10.1080/135048699334528}

[11] C. W. Oosterlee, "On Multigrid for Linear Complementarity Problems with Application to American-Style Options," Electronic Transactions on Numerical Analysis, Vol. 15, 2003, pp. 165-185.

http://etna.mcs.kent.edu/vol.15.2003/pp165-185.dir/pp16 5-185.pdf

[12] S. Ikonen and J. Toivanen, "Componentwise Splitting Methods for Pricing American Options under Stochastic Volatility," International Journal of Theoretical and Applied Finance, Vol. 10, No. 2, 2007, pp. 331-361. doi:10.1142/S0219024907004202

[13] J. M. Harrison and D. M. Kreps, "Martingales and Arbitrage in Multiperiod Securities Markets," Journal of Economic Theory, Vol. 20, 1979, pp. 381-408. doi:10.1016/0022-0531(79)90043-7

[14] M. Chernov and E. Ghysels, "A Study towards a Unified Approach to the Joint Estimation of Objective and Risk

\section{Appendix}

The conditional characteristic function of the state vector $\left(s_{T}, v_{T}\right)$ is

$$
\begin{aligned}
& \psi\left(\zeta_{1}, \zeta_{2} ; s_{t}, v_{t}, \tau\right) \\
= & \exp \left[p\left(\tau ; \zeta_{1}, \zeta_{2}\right)+q\left(\tau ; \zeta_{1}, \zeta_{2}\right) v_{t}+i \zeta_{1} s_{t}\right],
\end{aligned}
$$

where complex-valued functions $p\left(\tau ; \zeta_{1}, \zeta_{2}\right)$ and $q\left(\tau ; \zeta_{1}, \zeta_{2}\right)$ are defined below.

In particular, if $\gamma \neq 0$,

$$
\begin{aligned}
p\left(\tau ; \zeta_{1}, \zeta_{2}\right) & =\tau(r-\delta-\alpha \rho / \gamma) i \zeta_{1} \\
& +\frac{\alpha}{\gamma^{2}}\left[\tau \beta+\tau \sqrt{A\left(\zeta_{1}\right)}+2 \cdot \ln \frac{B\left(\zeta_{1}, \zeta_{2}\right)+1}{B\left(\zeta_{1}, \zeta_{2}\right) e^{\tau \sqrt{A\left(\zeta_{1}\right)}}+1}\right]
\end{aligned}
$$

and
Neutral Measures for the Purpose of Options Valuation," Journal of Financial Economics, Vol. 56, No. 3, 2000, pp. 407-458. doi:10.1016/S0304-405X(00)00046-5

[15] T. W. Epps, "Option Pricing under Stochastic Volatility with Jumps," Unpublished manuscript, University of Virginia, 2004.

[16] J. Gil-Pelaez, "Note on the Inversion Theorem," Biometrika, Vol. 38, No. 3-4, 1951, pp. 481-482. http://www.jstor.org/stable/2332598

[17] R. C. Merton, "Theory of Rational Option Pricing," Bell Journal of Economics and Management Science, Vol. 4, No. 1, 1973, pp. 141-183. doi:10.2307/3003143

[18] W. H. Press, S. A. Teukolsky, W. T. Vetterling and B. P. Flannery, "Numerical Recipes in Fortran 77: The Art of Scientific Computing," 2nd Edition (Reprint with Corrections), Cambridge University Press, Cambridge, MA, 2001.

$$
\begin{aligned}
& q\left(\tau ; \zeta_{1}, \zeta_{2}\right) \\
= & \frac{1}{\gamma^{2}} \times\left[\beta-\rho \gamma i \zeta_{1}-\sqrt{A\left(\zeta_{1}\right)} \frac{B\left(\zeta_{1}, \zeta_{2}\right) \mathrm{e}^{\tau \sqrt{A\left(\zeta_{1}\right)}}-1}{B\left(\zeta_{1}, \zeta_{2}\right) \mathrm{e}^{\tau \sqrt{A\left(\zeta_{1}\right)}}+1}\right],
\end{aligned}
$$

where

$$
A\left(\zeta_{1}\right)=\gamma^{2}\left(1-\rho^{2}\right) \zeta_{1}^{2}+\left(\gamma^{2}-2 \rho \gamma \beta\right) i \zeta_{1}+\beta^{2}
$$

and

$$
B\left(\zeta_{1}, \zeta_{2}\right)=-\frac{\rho \gamma i \zeta_{1}-\beta-\sqrt{A\left(\zeta_{1}\right)}+\gamma^{2} i \zeta_{2}}{\rho \gamma i \zeta_{1}-\beta+\sqrt{A\left(\zeta_{1}\right)}+\gamma^{2} i \zeta_{2}}
$$

The case of $\gamma=0$ is not particularly interesting because it rules out stochastic volatility. Expressions for the functions $p$ and $q$ in that case and all derivations can be found in Zhylyevskyy (2010) [7]. 